\title{
Robust Text Classifier for Classification of Spam E-Mail Documents with Feature Selection Technique
}

\author{
Akhilesh Kumar Shrivas ${ }^{1 *}$, Amit Kumar Dewangan², Samrendra Mohan Ghosh ${ }^{2}$ \\ ${ }^{1}$ Department of CSIT, Guru Ghasidas Vishwavidyalaya, Bilaspur, Chhattisgarh 495001, India \\ ${ }^{2}$ Computer Science Engineering, Dr. C. V. Raman University, Bilaspur, Chhattisgarh 495001, India
}

Corresponding Author Email: akhilesh.mca29@gmail.com

https://doi.org/10.18280/isi.260502

Received: 13 September 2021

Accepted: 13 October 2021

\section{Keywords:}

spam e-mail, classification, preprocessing, random forest (RF), feature selection technique (FST)

\begin{abstract}
E-mails are an effective medium for sending information in various modes like text, audio, video, etc. from one person to another. Spam e-mail is a junk e-mail that unnecessary wastage memory space, wasting time to delete and maintain e-mails in the mailbox. The contribution of this research work is to develop a robust and computational efficient classifier that classifies the spam e-mail and ham e-mail documents. This paper analyzes and validates the spam e-mails documents using different data mining-based classification techniques. The most importance of this research work is to select the best classifier with reduce feature subset of datasets that achieve better accuracy compared to other existing classifiers. We have collected six types of Enron datasets and prepared the last seven Enron datasets that combine all these six Enron datasets. Then, filtering the datasets with the help of the WEKA data mining tool. In the first step, we perform preprocessing the datasets and remove all the irrelevant words from the datasets. We have used different classifiers like Nave Bayes, J48, Random Forest, Random Tree, and Adaboosting to analyze and classify ham and spam e-mails documents. We also compare the performance of the classifier in terms of accuracy where Random Forest gives better accuracy with all seven Enron datasets. Finally, we have used the SymmetricalUncert feature selection technique to make the optimized dataset with a reduced feature subset. The suggested Random Forest classifier gives $98.73 \%$ of accuracy with reduced features of Enron datasets.
\end{abstract}

\section{INTRODUCTION}

E-mail is an effective and efficient communication medium to personal or official for any organization. One of the types of e-mail is the spam e-mail. Spam e-mail is a junk e-mail that is not necessary to harmful for users but it contains the unwanted details and send to the e-mail users by spammers. The first spam e-mail was generated on 3 may 1978 to several thousands of users on ARPANET sent by Gary Thuerk [1].

Sometimes many organizations or any unauthentic person use mails to sell the products, provide attractive offers related to products, send URLs, or any kind of offer greed to users such mails are called spam e-mails. There is no charge payable to sending e-mails so the person/organization sends the bulk number of e-mails to a different recipient. Spam e-mails are helpful to sell products and steal susceptible information. They use this information to involve users in any criminal activity or information to gain financial transactions. We collect this vital and sensitive information of people through spam e-mails, so spam e-mails are very deadly in today's computer age. Spam e-mail is a severe challenge because computer usage is becoming very fast in people today. But even today, people are not as knowledgeable as they should be, so we must face severe spam e-mail challenges very carefully. According to Kaspersky lab report, average spam e-mail traffic was $46.56 \%$, in Q2, 2021 which is grow up 0.89\% against the Q1, 2021 [2].
There are many ways like machine learning and classification to face difficulties like spam e-mails. Many researchers are currently using marching learning and data mining-based classification techniques like decision tree, naive bayes, support vector machine etc. as classifiers for classification of spam e-mail documents. The researchers are also using various evolutionary techniques like genetic algorithm, particle swarm optimization, principle component analysis to reduce the feature space of dataset.

Many researchers proposed various models and techniques to prevent spam e-mails. We analyzed those techniques and proposed a model that uses classification and feature selection techniques (FSTs). Classification is very effective techniques by which we can classify spam e-mails. The classification techniques can quickly point out the spam e-mails documents, and also increase accuracy of model by with FSTs. This work even more effectively and efficiently for reorganization and classification of spam e-mail and ham e-mail documents.

The remaining part of this research work as given where section 2 explores the review of literature related to spam email classification, section 3 examines the framework of spam e-mail documents classification using the proposed algorithm and also explore the different methods and materials have used in this paper, section 4 explores the experimental results, section 5 analyzes the result, and finally section 6 concludes the research work and also gives future direction. 


\section{LITERATURE REVIEW}

The literature review is an important section of a research paper. This section explored the research work done by different authors related to classification of spam e-mails documents. The authors [3] used spam e-mails and websites to study the detection of spam and also recognized the effectiveness of the Negative Selection Algorithm (NSA). NSA gave a high accuracy rate and low error rate. The authors $[4,5]$ used a number of papers related to spam e-mails in which machine learning techniques are useful to detect spam e-mails. In another paper, the authors [6] used the text semantic analysis method to classify spam and non-spam e-mails. The proposed method achieved better accuracy as compared to other methods. In this paper, the authors [7] used the removereplace feature selection technique (RRFST) to remove the features from dataset and achieved better accuracy with the proposed algorithm compared to others. The authors [8] used a novel spam-filtering technique which was based on analyzing the e-mail headers. They used the Hidden Markov Models (HMMs) for analyzing the header structure of e-mails and create a spam detection system. The authors [9] proposed the ALO-Boosting method to classify spam e-mails. In this method, ALO was used for finding the optimum feature subset which gave to boosting algorithm to help for better classification. According to the authors [10], irrelevant messages played a significant role in digital investigations. This message provides a lot of important information for spam e-mails' digital investigation. The authors [11] proposed an efficient algorithm to detect the threads and spam e-mails using the text analytics methodology with the help of e-mail spam corpus. It used the text keyword matching technique with the corpus to classify the spam and it prevents irrelevant mails in the inbox. This paper [12] used the artificial bee colony algorithm with a logistic regression classification model to identify spam e-mails. They used three different publicly available datasets to check their model ability and also compared the performance which is better than the available models. They used feature selection and wrapped methods to develop the technique. The authors [4] proposed the optimization technique to detect spam e-mails. They used the K-nearest neighbors algorithm with distance matrix Euclidean, Manhattan, and Chebyshev to classify the spam emails, then used different bio-inspired optimization techniques to classify the spam e-mails and achieved better accuracy. The authors [4] proposed a novel approach to classify the spam emails had three steps. In the first step, they used TFDCR FST, the second step described an incremental dynamic model to classify the dataset, and the last third step used the heuristic function to provide a strong ability to recognize the coming emails. The authors [13] used a bi-language e-mail text dataset to classify and create a cluster. NGram technique used and achieved better classification results. This paper [14] proposed the GA and RWN technique to detect spam e-mails and also implanted automatic feature detection techniques for better classification. The authors [15] proposed the review paper and read many research papers carefully and extract seven search strategies and got important conclusions related to approaches and techniques. In this paper, the author [16] worked with two real-life datasets to detect opinion spam with the help of a complex probabilistic graph classification approach. They used the neural network technique along with a heterogeneous graph to connect the nodes for concluding about the opinion spam. The main theme of this paper [17] is to classify spam and phishing e-mails. They used the body structure of the email and applies deep-learning and FSTs to identify the emails into different categories. The authors [18] used both a supervised regular expression pattern matching technique and an unsupervised K-mean machine learning algorithm to identify the insider threads using analysis of the structure of the e-mails. The author [19] used ID3 and Hidden Markov model to identify spam e-mails and achieved better performance to detect spam e-mails.

This section discussed about SMS classification and twitter spam classification. The authors [20] used two experiments for identifying SMS spam. In the first experiment, they used the Bayesian network classifier method along with the costsensitive technique, and in the second experiment, they compared the performance of the proposed technique with existing techniques. The author [21] proposed the MWOASPD hybrid technique to detect and classify the tweeter spams.

This section explored the classification of ham and spam documents with Enron dataset. The authors [22] observed security threads about big data in studies. They used the Enron dataset (contains spam and ham documents) to collect a conclusion related to the security issue of big data and also studied in students how they react to spam e-mails. In this research [23], the author preferred the Enron people assignment dataset to create the model and provided the proper recipient. In this paper [24], the author generated a synthetic dataset with the help of the Enron e-mail dataset along with the STDG simulator application to detect threads and knowledge discoveries.

\section{PROPOSED FRAMEWORK AND METHODOLOGY}

This section discussed the architecture of the proposed framework and methodology. Figure 1 shows the proposed architecture for the classification of spam e-mail. In this architecture, the Enron datasets are collected from the Kaggle repository, and then applied different preprocessing techniques for smoothing the datasets; hence model can achieve better accuracy. The partition of datasets into training and testing is one of the essential steps of the data classification process. This research work has used 10-fold cross-validation for the partition of a dataset into training and testing. We have applied the training and testing of spam and ham messages into different classifiers like Naïve Bayes, J48, RF, Random Tree, and AdaBoosting and evaluate the performance of classifiers in terms of accuracy where RF achieved better accuracy with all seven types of Enron datasets. The RF gained the highest as $98.68 \%$ of accuracy with the combined Enron dataset. Finally, we have applied the ChiSquare FST on the combined Enron dataset and reduce the features of datasets. The recommended RF gives the highest as $98.74 \%$ of accuracy with reduced features of the combined Enron dataset. 


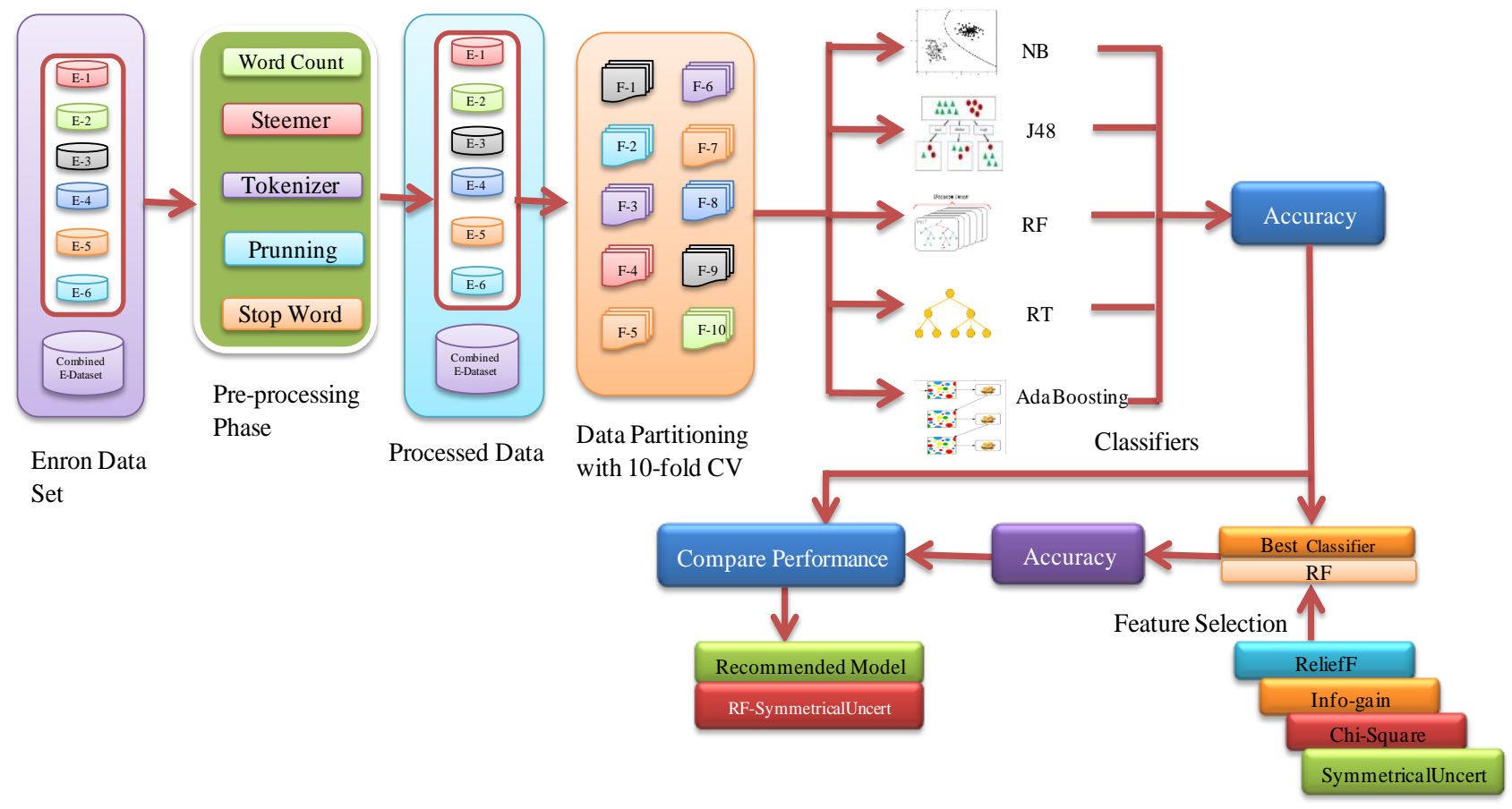

Figure 1. Framework for spam e-mail documents classification

The pseudo code of proposed framework as given below:

\section{Start}

Input:

Enron Dataset (Enron1, Enron2, Enron3, Enron4, Enron5, Enron6, Combined Enron Dataset).

The dataset contains spam and ham e-mails documents.

\section{Output:}

Performance Measures $=\mathrm{PM}=\{$ Acc, Sen, Spc, Pr, Fs $\}$

Mbest $=$ Best model, ReducedF_Enron $=$ Enron dataset with reduced features.

where, Ac $=$ Accuracy, Sen $=$ Sensitivity, Spec=Specificity, $\operatorname{Pr}=$ Precision, Fs=F-score,

1. Read Enron datasets

- For input $\mathrm{i}=1$ to total number of datasets:

- $\quad$ Read Enron(i)

- For input $\mathrm{j}=1$ to length of datasets:

- do word count and remove stop words, Stemming

- $\quad$ words, tokens and prune dataset

- $\quad$ Record words along with their frequency

- $\quad$ End of inner For

- $\quad$ Return Preprocessed_Enron(i)

- $\quad$ End For

2. Read Preprocessed_Enron datasets

- $\quad$ For input $\mathrm{k}=1$ to total number of datasets:

- $\quad \mathrm{M}(\mathrm{k})=$ Preprocessed_Enron $(\mathrm{k}) \leftarrow\{$ Naïve Bayes,

J48, RF, RT,AdaBoosting\}

- $\quad \operatorname{Mbest}(\mathrm{k})=\operatorname{Compare}\{(\mathrm{M}(\mathrm{k}) \leftarrow \mathrm{Ac})\}$

- $\quad$ End For

- $\quad$ Mbest $\rightarrow(R F)=\{$ Ac, Sen, Spec, Pr, Fs $\}$

3. Read Mbest $\rightarrow(\mathrm{RF})$

- For input $\mathrm{l}=1$ to length of datasets:educedF_Enron = $\{$ Preprocessed_Enron $\} \leftarrow\{$ Chi-Square, Infogain, ReliefF, SymmetricalUncert $\}$

- $\quad$ Record words along with their frequency

- $\quad \mathrm{M}(\mathrm{l})=$ ReducedF_Enron $\leftarrow\{\mathrm{RF}\}$

- $\quad \operatorname{Mbest}(1)=\operatorname{Compare}\{\mathrm{M}(1) \leftarrow \mathrm{Ac}\}$
- $\quad$ End For

- $\quad$ Mbest $(1)=\{$ Ac, Sen, Spec, Pr, Fs $\}$

End.

\subsection{Enron dataset}

Dataset is a significant input for any research. In this research, we have used the Enron dataset collected from the UCI repository. We have collected six different types of Enron datasets. The last seven Enron dataset named as combined Enron dataset is prepared with a combination of collecting all six Enron datasets. Each Enron dataset contains set of the emails including spam and ham e-mails documents generated by employees of the Enron Corporation. The main reason for selecting Enron dataset is to develop the robust model that is able to classify the ham and spam documents with high accuracy. The total instances present in each Enron dataset has displayed in a Table 1 .

Table 1. Description of Enron datasets

\begin{tabular}{cccc}
\hline Datasets & $\begin{array}{c}\text { Ham- } \\
\text { instance }\end{array}$ & $\begin{array}{c}\text { Spam- } \\
\text { instance }\end{array}$ & $\begin{array}{c}\text { Total } \\
\text { instances }\end{array}$ \\
\hline Enron-1 & 3671 & 1500 & 5172 \\
Enron-2 & 4361 & 1496 & 5857 \\
Enron-3 & 4012 & 1500 & 5512 \\
Enron-4 & 1500 & 4499 & 5999 \\
Enron-5 & 1500 & 3675 & 5175 \\
Enron-6 & 1500 & 4500 & 6000 \\
Combine & 16383 & 16383 & 32766 \\
Enron & & & \\
\hline
\end{tabular}

\subsection{Preprocessing}

Preprocessing is a very crucial step for the text mining experiment. The big challenge about text mining is to convert unstructured data into structured data. There are several steps involved in transforming unstructured data into structured data. 
We follow a number of steps like word count, stemming, tokenization, pruning, and stop word. Word counts measures the total number of words presents in the documents. Stemming means finding the root of words. Tokenization is the process of turning a meaningful piece of data. Pruning is a way to remove unimportant words to improve the structure data and finally use stop words from removing commonly used words like articles (a, an, the, is, etc.), preposition, etc.

\subsection{Data partition}

The data partition is a technique to partition the data into training and testing sets. Cross-validation [25] is a data partition technique used to analyze the performance of the machine learning techniques. We have used a 10-fold crossvalidation technique to create a partition of the dataset into ten equal parts, randomly selecting one part of the dataset as a testing dataset. The remaining 9 part is for the training dataset. This iteration occurs ten times to perform the analysis of the algorithm.

\subsection{Classification techniques}

There are different classification algorithm resides in the WEKA tools (http:// www.cs.waikato.ac.nz/ ml/weka/). This algorithm provides various principles to classify the text data. There are different algorithm likes Naive Bayes, J48, RF, Random Tree, and Adaboosting.

\subsubsection{Naïve Bayes}

Naïve Bayes [26] classifier strictly follows the Bayes' theorem principle. Bayes theorem works the conditional probability principle. It used posterior probability in this kind of method. Naive Bayes is useful for the large size of the dataset.

\section{$3.4 .2 \mathrm{~J} 48$}

$\mathrm{J} 48$ [27] is also known as C4.5. The C4.5 algorithm is the successor of ID3 (iterative Dichotomiser). C4.5 follows the non-backtracking approach of the greedy method in which the decision tree builds via top-down recursive and it also follows the divide-and-conquer methodology. All the tuples are associated with the class labels. We have used the training set to build the tree using the recursively partitioned method.

\subsubsection{Random Forest}

Random Forest (RF) [28] follows the principle of a supervised machine learning algorithm. As the name suggests, a RF comprises numerous decision trees. The model prediction depends upon the class of the most vote which come from the decision tree, that's why random forests follow the wisdom of crowds. RF is useful for large-size datasets and has a large number of input features. The classification problem can handle by either the Gini-index or the entropy in a RF.

$$
\text { Gini }=1-\sum_{k=0}^{n}(X i)^{k}
$$

When we use the entropy to find the decision node in a random tree can be expressed like

$$
\text { Entropy }=\sum_{k=0}^{n}-X i * \log 2(X i)
$$

$\mathrm{Xi}$ represents the relative frequency of the class that observes in the dataset, and $\mathrm{n}$ represents the number of classes.

\subsubsection{Random tree}

The random tree [26, 29] has been introducing by Leo Breiman and Adele Cutler. A random tree algorithm is helpful to solve both regression and classification problems. The random tree belongs to the supervised learning group. It is an ensemble learning algorithm that generates many individual learners. It enlists a bagging scheme to generate a random set of data for building a decision tree.

\subsubsection{Adaboosting}

The abbreviation of Adaptive Boosting [30] is AdaBoost. The AdaBoost algorithm was the first algorithm created for binary classification. With the help of the AdaBoost algorithm easily increase the performance of any machine learning algorithm. It is very helpful for weak learners. The model which used the AdaBoost algorithm attains better accuracy as compared to other classification problems. The equation is represented by

$$
\mathrm{F}(\mathrm{x})=\operatorname{Sign}\left(\sum_{k=1}^{K} W k * F k(x)\right)
$$

where, Fk represents the Kth weak classifier and $\mathrm{Wk}$ represents the corresponding weight.

\subsection{Feature selection}

Feature selection is a technique that selects the most valuable feature among all features. In this technique, we select all the features that have a significant effect and remove all the features that do not have a significant effect. In this research, we use the FST followed by the ranker method.

Chi-square [31] used the relation between feature and category of words. The feature means the occurrence of frequency of feature and category means the probability of occurrence of category. We find the correlation between feature and category, if they are dependent then find the level of correlation between them, and if independent then we do not apply the FST. SymmetricalUncert [32] FST evaluates the worth of an attribute by measuring the symmetrical uncertainty with respect to the class. This method follows the ranking technique to calculate the rank of the feature. It can remove the feature whose value is less than the threshold value. Information Gain (IG) [33] is an entropy-based FST, followed by the ranker method. IG is defined as the amount of information provided by the feature items for the text category. IG is calculated by how much of the term can be used for the classification of information, in order to measure the importance of lexical items for the classification. ReliefF [34] FST was the extension of the Relief feature selection algorithm in 1994. ReliefF can handle the classification of multi-class data. ReliefF selects the random sample from the training set. After that, it finds out $\mathrm{k}$ near hits from the same class and $\mathrm{k}$ near misses from each different class. In this way, it updates the weight of the feature and repeats this process $\mathrm{n}$ times to increase the weights of all features.

\section{EXPERIMENTAL RESULTS}

In this section, we present the results related to our experiment. We accomplished the experimental work using the WEKA tool in Windows 10 operating system environment. In this research work, we have used six Enron datasets to find the classification accuracy between ham and spam e-mails. 
This experiment is divided into two parts: In the first part, we take six different Enron datasets, and the second part combines all six datasets and creates one signal combined dataset. Table 2 shows the number of instances of Enron datasets. We have applied pre-processing techniques like word count, steamer, tokenizer, pruning, and stop word to remove the unimportant words from both kinds of datasets as shown in Table 3. Table 3 shows the remaining words after applying preprocessing technique in datasets. Figure 2 is the graphical representation of the words related to all datasets. After that, we apply machine learning algorithms like Naïve Bayes, J48, RF, Random Tree, and Adaboosting to get accuracy which is shown in Table 4. Table 4 shows that the accuracy of classifiers like Naïve Bayes, J48, RF, Random Tree, and Adaboosting with each dataset and also get one more conclusion that RF achieves the highest accuracy with each dataset. Figure 3 is the graphical representation of accuracy related to each dataset. In Table 5, we represent the confusion matrix related to the highest accuracy achieved by the RF algorithm with the combined Enron dataset. We have also calculated different performance measures like true positive rate (TPR), false positive rate (FPR), precision, specificity, and F-measures, which are represented in Table 6. In the next section, we apply the FSTs like ReliefF, Info-gain, Chi-square, and SymmetricalUncert followed by the ranker method in the combined Enron dataset with RF machine learning algorithm using a 10 fold cross-validation data partition method and achieved better accuracy with 10000 features. The RF achieved an accuracy of $98.73 \%$ is the highest among other accuracy with SymmetricalUncert FSTs in the case of the combined Enron dataset as shown in Table 7. We show the confusion matrix and different performance measures of RF in
Table 8 and Table 9 respectively. Figure 4 shows that the performance measures of the best classifier RF with different FSTs.

The above Table 3 and Figure 2 show that the relevant numbers of words in datasets after applying preprocessing techniques like wordcounts, steamer, tokenizer, pruning and stop word.

Table 4 and Figure 3 show that the accuracy of different classifiers with different Enron datasets where Random Forest classifiers gives best accuracy compared to others.

Table 9 and Figure 4 show that the various performance measures of Random Forest classifiers with different FSTs. Finally, this research work has compared the performance of our suggested RF classifier with SymmetricalUncert FST to the existing models where suggested RF achieved better accuracy compared to others. In the base paper [3], the author used a combination of six Enron datasets and got $98.57 \%$ of accuracy but our suggested classifier RF achieved $98.68 \%$ of accuracy, and at the same time, we have applied SymmetricalUncert FST on a combined Enron dataset where RF got $98.73 \%$ of accuracy with reduced feature subsets.

Table 2. Number of instances of Enron datasets

\begin{tabular}{cc}
\hline Name of datasets & Number of instances \\
\hline Enron-1 & 5172 \\
Enron-2 & 5857 \\
Enron-3 & 5512 \\
Enron-4 & 5999 \\
Enron-5 & 5175 \\
Enron-6 & 6000 \\
Combined Enron & 32766 \\
\hline
\end{tabular}

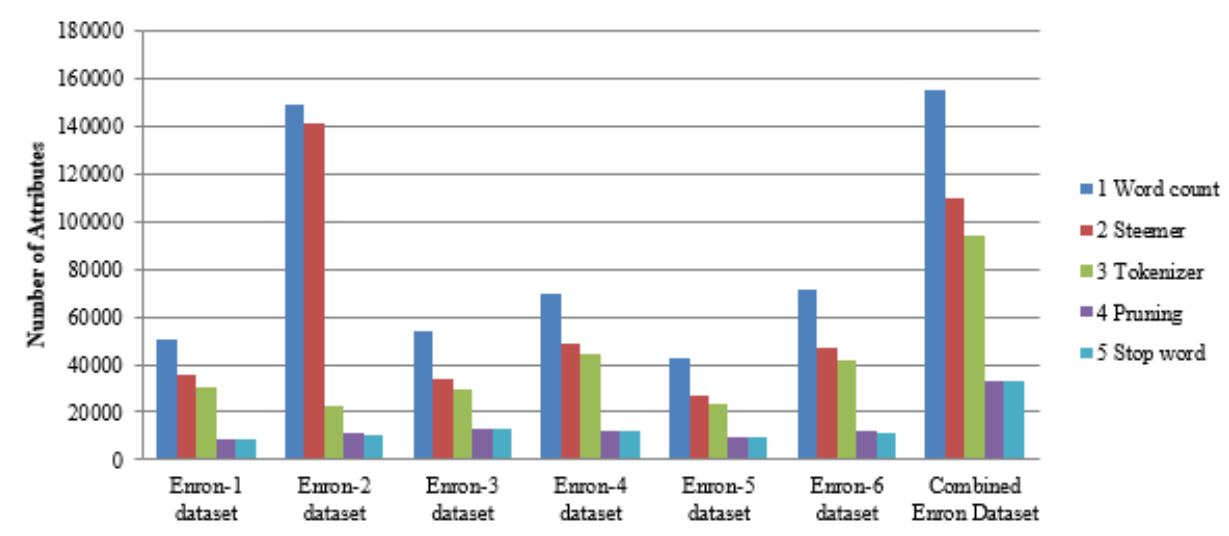

Figure 2. Graphical representation of preprocessed datasets

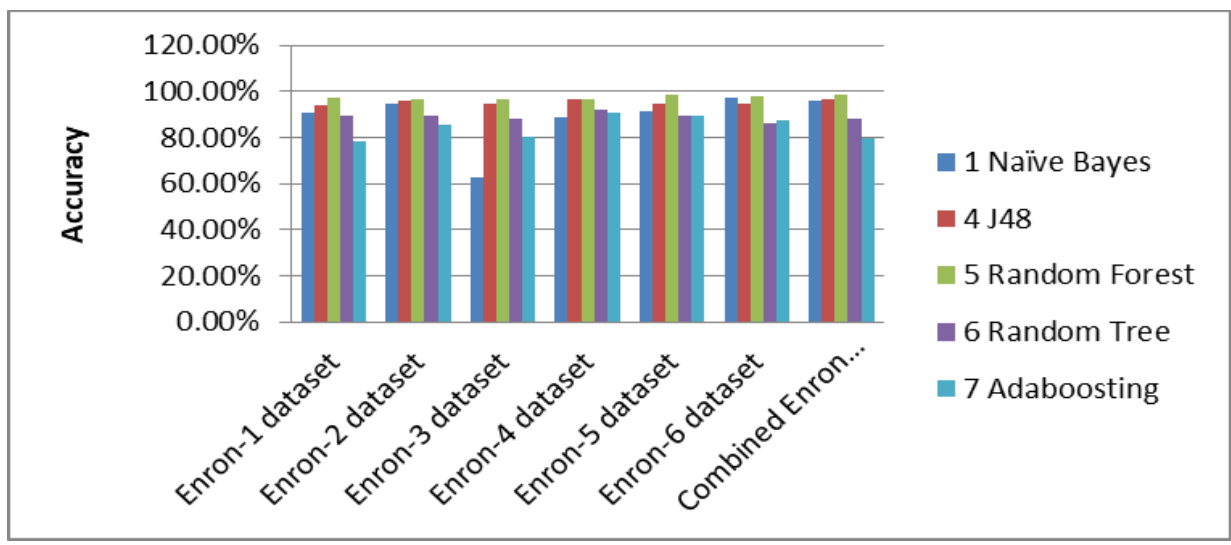

Figure 3. Graphical representation of classifiers accuracy with different Enron datasets 


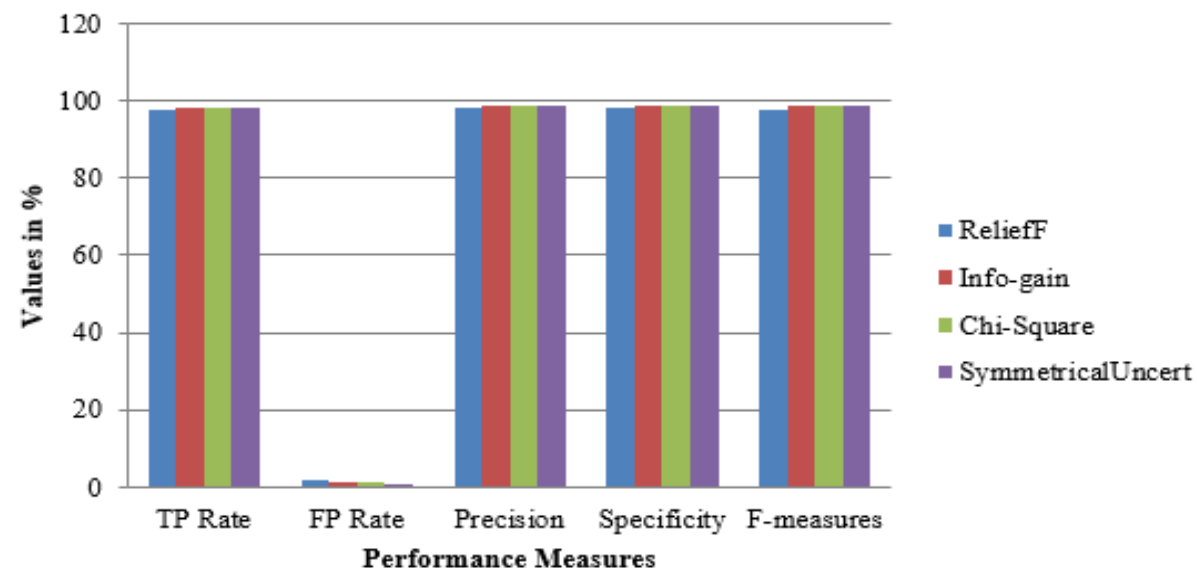

Figure 4. Performance measures of best classifier RF with FST in case of combined Enron dataset

Table 3. Preprocessing of Enron datasets

\begin{tabular}{ccccccccc}
\hline \multirow{2}{*}{$\begin{array}{c}\text { Sr. } \\
\text { No. }\end{array}$} & $\begin{array}{c}\text { Preprocessing } \\
\text { steps }\end{array}$ & $\begin{array}{c}\text { Enron-1 } \\
\text { dataset }\end{array}$ & $\begin{array}{c}\text { Enron-2 } \\
\text { dataset }\end{array}$ & $\begin{array}{c}\text { Enron-3 } \\
\text { dataset }\end{array}$ & $\begin{array}{c}\text { Enron-4 } \\
\text { dataset }\end{array}$ & $\begin{array}{c}\text { Enron-5 } \\
\text { dataset }\end{array}$ & $\begin{array}{c}\text { Enron-6 } \\
\text { dataset }\end{array}$ & $\begin{array}{c}\text { Combined Enron } \\
\text { Dataset }\end{array}$ \\
\hline 1. & Word count & 50557 & 148914 & 53872 & 69528 & 42285 & 71597 & 155115 \\
2. & Stemmer & 35623 & 141393 & 33864 & 48969 & 27335 & 47158 & 109459 \\
3. & Tokenizer & 30852 & 22214 & 29726 & 44176 & 23694 & 41838 & 94498 \\
4. & Pruning & 8763 & 10850 & 13030 & 12219 & 9333 & 11811 & 33517 \\
5. & Stop word & 8529 & 10612 & 12788 & 11980 & 9100 & 11575 & 33264 \\
\hline
\end{tabular}

Table 4. Accuracy of the classifiers with Enron datasets

\begin{tabular}{ccccccccc}
\hline $\begin{array}{c}\text { Sr. } \\
\text { No. }\end{array}$ & Classifier & $\begin{array}{c}\text { Enron-1 } \\
\text { dataset }\end{array}$ & $\begin{array}{c}\text { Enron-2 } \\
\text { dataset }\end{array}$ & $\begin{array}{c}\text { Enron-3 } \\
\text { dataset }\end{array}$ & $\begin{array}{c}\text { Enron-4 } \\
\text { dataset }\end{array}$ & $\begin{array}{c}\text { Enron-5 } \\
\text { dataset }\end{array}$ & $\begin{array}{c}\text { Enron-6 } \\
\text { dataset }\end{array}$ & $\begin{array}{c}\text { Combined Enron } \\
\text { Dataset }\end{array}$ \\
\hline $\mathbf{1}$ & Naïve Bayes & $90.93 \%$ & $94.83 \%$ & $62.94 \%$ & $88.78 \%$ & $91.49 \%$ & $97.12 \%$ & $96.15 \%$ \\
$\mathbf{2}$ & $\begin{array}{c}\text { J48 } \\
\mathbf{3}\end{array}$ & $93.72 \%$ & $95.99 \%$ & $94.92 \%$ & $96.38 \%$ & $94.38 \%$ & $94.43 \%$ & $96.38 \%$ \\
$\mathbf{4}$ & $\begin{array}{c}\text { Random Forest } \\
\text { (RF) }\end{array}$ & $\mathbf{9 7 . 5 3 \%}$ & $\mathbf{9 6 . 3 8 \%}$ & $\mathbf{9 6 . 8 3 \%}$ & $\mathbf{9 6 . 4 8} \%$ & $\mathbf{9 8 . 5 9 \%}$ & $\mathbf{9 7 . 9 7 \%}$ & $\mathbf{9 8 . 6 8 \%}$ \\
$\mathbf{5}$ & Random Tree & $89.23 \%$ & $89.57 \%$ & $88.28 \%$ & $92.12 \%$ & $89.60 \%$ & $86.45 \%$ & $87.92 \%$ \\
\hline
\end{tabular}

Table 5. Confusion matrix of best RF classifier with Enron datasets

\begin{tabular}{cccccccccccccc}
\hline \multirow{2}{*}{$\begin{array}{c}\text { Actual vs. } \\
\text { Predicted }\end{array}$} & \multicolumn{2}{c}{$\begin{array}{c}\text { Enron-1 } \\
\text { dataset }\end{array}$} & \multicolumn{2}{c}{$\begin{array}{c}\text { Enron-2 } \\
\text { dataset }\end{array}$} & \multicolumn{2}{c}{$\begin{array}{c}\text { Enron-3 } \\
\text { dataset }\end{array}$} & \multicolumn{2}{c}{$\begin{array}{c}\text { Enron-4 } \\
\text { dataset }\end{array}$} & \multicolumn{2}{c}{$\begin{array}{c}\text { Enron-5 } \\
\text { dataset }\end{array}$} & \multicolumn{2}{c}{$\begin{array}{c}\text { Enron-6 } \\
\text { dataset }\end{array}$} & \multicolumn{2}{c}{$\begin{array}{c}\text { Combine Enron } \\
\text { dataset }\end{array}$} \\
& Ham & Spam & Ham & Spam & Ham & Spam & Ham & Spam & Ham & Spam & Ham & Spam & Ham \\
Spam
\end{tabular}

Table 6. Performance measures of best RF classifier with different Enron datasets

\begin{tabular}{cccccccc}
\hline \multirow{2}{*}{ Name of Measures } & & \multicolumn{7}{c}{ Datasets } \\
& Enron-1 & Enron-2 & Enron-3 & Enron-4 & Enron-5 & Enron-6 & Combined Enron \\
\hline TP Rate & 98.09 & 99.68 & 99.90 & 85.93 & 95.27 & 91.93 & 98.54 \\
FP Rate & 3.87 & 13.24 & 11.40 & 0 & 0.05 & 0.02 & 1.18 \\
Precision & 98.42 & 95.64 & 95.91 & 100 & 99.86 & 99.93 & 98.81 \\
Specificity & 96.13 & 86.76 & 88.6 & 100 & 99.95 & 99.98 & 98.82 \\
F-measures & 98.25 & 97.62 & 97.86 & 92.43 & 97.51 & 95.76 & 98.67 \\
\hline
\end{tabular}

Table 7. Accuracy of RF with FSTs with Combined Enron datasets

\begin{tabular}{cc}
\hline Feature Selection Techniques & Accuracy in \% \\
\hline ReliefF & 97.88 \\
Info-gain & 98.68 \\
Chi-Square & 98.71 \\
SymmetricalUncert & 98.73 \\
\hline
\end{tabular}


Table 8. Confusion matrix RF with FSTs in case of Combined Enron datasets

\begin{tabular}{ccccccccc}
\hline Actual & \multicolumn{2}{c}{ ReliefF } & \multicolumn{2}{c}{ Info-gain } & \multicolumn{2}{c}{ Chi-Square } & \multicolumn{2}{c}{ SymmetricalUncert } \\
Vs. Predicted & Ham & Spam & Ham & Spam & Ham & Spam & Ham & Spam \\
\hline Ham & 15976 & 407 & 16118 & 265 & 16126 & 257 & 16122 & 261 \\
Spam & 288 & 16095 & 167 & 16216 & 165 & 16218 & 156 & 16227 \\
\hline
\end{tabular}

Table 9. Performance measures of RF with FSTs in case of Combined Enron datasets

\begin{tabular}{ccccc}
\hline Name of attributes & ReliefF & Info-gain & Chi-Square & SymmetricalUncert \\
\hline TP Rate & 97.52 & 98.38 & 98.43 & 98.41 \\
FP Rate & 1.76 & 1.02 & 1.01 & 0.95 \\
Precision & 98.23 & 98.97 & 98.99 & 99.04 \\
Specificity & 98.24 & 98.98 & 98.99 & 99.05 \\
F-measures & 97.87 & 98.67 & 98.71 & 98.72 \\
\hline
\end{tabular}

\section{CONCLUSION AND FEATURE WORKS}

Classification of spam e-mail documents with high accuracy is a very challenging task for researchers. In this paper, we have prepared a model related to a spam e-mails documents classification. We have used seven Enron datasets, which are Enron-1, Enron-2, Enron-3, Enron-4, Enron-5, Enron-6, and combined Enron datasets. This research work has used classification techniques to analyze the Enron dataset and classify the spam and ham e-mails documents. We have used Naïve Bayes, J48, RF, Random Tree, and AdaBoosting algorithms to develop a model. The RF classifier achieves the highest accuracy in all seven datasets compared to other algorithms. The RF classifier gives the highest $98.68 \%$ of accuracy in the case of the combined Enron dataset. Then, we apply the SymmetricalUncert FST on the combined Enron dataset and classify and analyzed it with a RF algorithm where RF achieves better accuracy as $98.73 \%$. Finally, we have suggested that RF with the SymmetricalUncert FST model is better for the classification of spam e-mails documents. In the future, we will develop a robust and computationally intelligent hybrid model which will give better accuracy compared to others in the field of spam filtering, phishing emails classification and different types of attacks. We will also develop new feature selection and optimization techniques which will reduce the irrelevant number of features (words) from dataset and achieve the better classification accuracy with a smaller number of features and less computational time.

\section{ACKNOWLEDGMENTS}

This research did not receive any specific grant from funding agencies in the public, commercial, or not-for-profit sectors. The authors would like to thank the editor and anonymous reviewers for their comments that help improve the quality of this work.

\section{REFERENCES}

[1] Weblink: https://en.wikipedia.org/wiki/History_of_email_spam, accessed on 22 July 2021.

[2] Kaspersky Lab Spam Report. (2021). https://securelist.com/spam-and-phishing-in-q22021/103548/, accessed on 20 Aug. 2021.

[3] Saleh, A.J., Karim, A., Shanmugam, B., Azam, S.,
Kannoorpatti, K., Jonkman, M., Boer, F.D. (2019). An intelligent spam detection model based on artificial immune system. Information.s Information, 10(6): 1-17. https://doi.org/10.3390/info10060209

[4] Batra, J., Jain, R., Tikkiwal, V.A., Chakraborty, A. (2021). A comprehensive study of spam detection in emails using bio-inspired optimization techniques. Int. J. Inf. Manag. Data Insights, 1(1): 1-13. https://doi.org/10.1016/j.jjimei.2020.100006

[5] Dada, E.G., Bassi, J.S., Chiroma, H., Abdulhamid,S.M., Adetunmbi, A.O., Ajibuwa, O.E. (2019). Machine learning for email spam filtering: Review, approaches and open research problems. Heliyon, 5(6): 1-23. https://doi.org/10.1016/j.heliyon.2019.e01802

[6] Saidani, N., Adi, K., Allili, M.S. (2020). A semanticbased classification approach for an enhanced spam detection. Comput. Secur., 94: 1-12. https://doi.org/10.1016/j.cose.2020.101716

[7] Hota, H.S., Shrivas, A.K., Hota, R. (2018). An ensemble model for detecting phishing attack with proposed remove-replace feature selection technique. Procedia $\begin{array}{llll}\text { Comput. } & \text { Sci., } & \text { 132: } & \text { 900-907. }\end{array}$ https://doi.org/10.1016/j.procs.2018.05.103

[8] Salcedo-Campos, F., Díaz-Verdejo, J., García-Teodoro, P. (2012). Segmental parameterisation and statistical modelling of e-mail headers for spam detection. Inf. Sci. (Ny)., 195: 45-61. https://doi.org/10.1016/j.ins.2012.01.022

[9] Naem, A.A., Ghali, N.I., Saleh, A.A. (2018). Antlion optimization and boosting classifier for spam email detection. Futur. Comput. Informatics J., 3(2): 436-442. https://doi.org/10.1016/j.fcij.2018.11.006

[10] Yu, S. (2015). Covert communication by means of email spam: A challenge for digital investigation. Digit. Investig., 13: 72-79. https://doi.org/10.1016/j.diin.2015.04.003

[11] Murugavel, U., Santhi, R. (2020). Detection of spam and threads identification in E-mail spam corpus using content based text analytics method. Materials Today: Proceedings, 33: 3319-3323. https://doi.org/10.1016/j.matpr.2020.04.742

[12] Dedeturk, B.K., Akay, B. (2020). Spam filtering using a logistic regression model trained by an artificial bee colony algorithm. Appl. Soft Comput. J., 91: 106229. https://doi.org/10.1016/j.asoc.2020.106229

[13] Alsmadi, I., Alhami, I. (2015). Clustering and classification of email contents. J. King Saud Univ. Comput. Inf. Sci., 27(1): 46-57. 
https://doi.org/10.1016/j.jksuci.2014.03.014

[14] Faris, H. (2019). An intelligent system for spam detection and identification of the most relevant features based on evolutionary Random Weight Networks. Inf. Fusion, 48:

67-83. https://doi.org/10.1016/j.inffus.2018.08.002

[15] Abayomi-Alli, O., Misra, S., Abayomi-Alli, A., Odusami, M. (2019). A review of soft techniques for SMS spam classification: Methods, approaches and applications. Eng. Appl. Artif. Intell., 86: 197-212. https://doi.org/10.1016/j.engappai.2019.08.024

[16] Liu, Y., Pang, B., Wang, X. (2019). Opinion spam detection by incorporating multimodal embedded representation into a probabilistic review graph. Neurocomputing, 366: 276-283. https://doi.org/10.1016/j.neucom.2019.08.013

[17] Rastenis, J., Ramanauskaite, S., Suzdalev, I., Tunaitytè, K., Janulevičius, J., Čenys, A. (2021). Multi-language spam/phishing classification by email body text: Toward automated security incident investigation. Electron., 10(6):

$1-10$. https://doi.org/10.3390/electronics10060668

[18] Michael, A., Eloff, J.H.P. (2019). A machine learning approach to detect insider threats in emails caused by human behaviours. Proceedings of the Thirteenth International Symposium on Human Aspects of Information Security \& Assurance (HAISA 2019), 34-49.

[19] Kumar, V., Monika, Kumar, P., Sharma, A. (2018). Spam email detection using ID3 algorithm and hidden Markov Model. 2018 Conf. Inf. Commun. Technol. CICT 2018, pp. 1-6 https://doi.org/10.1109/INFOCOMTECH.2018.872237 8

[20] Lim, L.P., Singh, M. (2020). Resolving the imbalance issue in short messaging service spam dataset using costsensitive techniques. J. Inf. Secur. Appl., 54: 1-10. https://doi.org/10.1016/j.jisa.2020.102558

[21] Krithiga, R., Ilavarasan, E. (2020). A reliable modified whale optimization algorithm based approach for feature selection to classify twitter spam profiles. Microprocess. Microsyst. https://doi.org/10.1016/j.micpro.2020.103451

[22] Zaki, T., Uddin, M.S., Hasan, M.M., Islam, M.N. (2017). Security threats for Big Data. Int. Conf. Res. Innov. Inf. Syst. ICRIIS, $\quad$ pp. https://doi.org/10.1109/ICRIIS.2017.8002481

[23] Rameshkumar, R., Bailey, P., Jha, A., Quirk, C., (2019). Assigning people to tasks identified in email: The EPA dataset for addressee tagging for detected task intent. Proceedings of the 2018 EMNLP Workshop W-NUT: The 4th Workshop on Noisy User-generated Text, Brussels, Belgium, pp. 28-32. https://doi.org/10.18653/v1/w18-6104

[24] Babalola, K.O., Jennings, O.B., Urdiales, E., Debardelaben, J.A. (2019). Statistical methods for generating synthetic email data sets. Proc. - 2018 IEEE
Int. Conf. Big Data, Big Data 2018, 3986-3990. https://doi.org/10.1109/BigData.2018.8622601

[25] Laorden, C., Santos, I., Sanz, B., Alvarez, G., Bringas, P.G. (2012). Word sense disambiguation for spam filtering. Electron. Commer. Res. Appl., 11(3): 290-298. https://doi.org/10.1016/j.elerap.2011.11.004

[26] Gupta, A., Mohan, K.M., Shidnal, S. (2018). Spam filter using naïve bayesian technique. Int. J. Comput. Eng. Res. 8(6): 2250-3005.

[27] Yadav, A.K., Chandel, S.S. (2015). Solar energy potential assessment of western Himalayan Indian state of Himachal Pradesh using J48 algorithm of WEKA in ANN based prediction model. Renew. Energy, 75: 675693. https://doi.org/10.1016/j.renene.2014.10.046

[28] Aulia, A., Jeong, D., Saaid, I.M., Kania, D., Shuker, M.T., El-Khatib, N.A. (2019). A random forests-based sensitivity analysis framework for assisted history matching. J. Pet. Sci. Eng., 181: 106237. https://doi.org/10.1016/j.petrol.2019.106237

[29] Kalmegh, S. (2015). Analysis of WEKA data mining algorithm reptree, simple cart and randomtree for classification of Indian News. Int. J. Innov. Sci. Eng. Technol., 2(2): 438-446.

[30] Mazini, M., Shirazi, B., Mahdavi, I. (2019). Anomaly network-based intrusion detection system using a reliable hybrid artificial bee colony and AdaBoost algorithms. J. King Saud Univ. - Comput. Inf. Sci., 31(4): 541-553. https://doi.org/10.1016/j.jksuci.2018.03.011

[31] Rachburee, N., Punlumjeak, W. (2015). A comparison of feature selection approach between greedy, IG-ratio, Chi-square, and mRMR in educational mining. Proc. 2015 7th Int. Conf. Inf. Technol. Electr. Eng. Envisioning Trend Comput. Inf. Eng. ICITEE 2015, 420424. https://doi.org/10.1109/ICITEED.2015.7408983

[32] Dai, J., Chen, J., Liu, Y., Hu, H. (2020). Novel multilabel feature selection via label symmetric uncertainty correlation learning and feature redundancy evaluation. Knowledge-Based Syst., 207: 106342. https://doi.org/10.1016/j.knosys.2020.106342

[33] Lei, S. (2012). A feature selection method based on information gain and genetic algorithm. Proc. - 2012 Int. Conf. Comput. Sci. Electron. Eng. ICCSEE 2012, 2: 355358. https://doi.org/10.1109/ICCSEE.2012.97

[34] Yang, F., Cheng, W., Dou, R., Zhou, N. (2011). An improved feature selection approach based on ReliefF and mutual information. 2011 Int. Conf. Inf. Sci. Technol. ICIST 2011, pp. 246-250. https://doi.org/10.1109/ICIST.2011.5765246

\section{NOMENCLATURE}

FST Feature Selection Technique

RF Random Forest

TP True Positive

FP False Positive 